\title{
Network Formation Game for Multi-Hop Wearable Communications over Millimeter Wave Frequencies
}

\author{
Qianqian Zhang ${ }^{1}$, Walid Saad ${ }^{1}$, Mehdi Bennis ${ }^{2}$, and Mérouane Debbah ${ }^{3,4}$ \\ ${ }^{1}$ Bradley Department of Electrical and Computer Engineering, Virginia Tech, Blacksburg, VA, USA, Emails: \{qqz93,walids\}@ vt.edu \\ ${ }^{2}$ Center for Wireless Communications-CWC, University of Oulu, Finland, Email:bennis@ee.oulu.fi \\ ${ }^{3}$ Mathematical and Algorithmic Sciences Lab, Huawei France R\&D, Paris, France, Email:merouane.debbah@huawei.com \\ ${ }^{4}$ Large Systems and Networks Group (LANEAS), CentraleSupélec, Université Paris-Saclay, 3 rue Joliot-Curie, 91192 Gif-sur-Yvette, France
}

\begin{abstract}
In this paper, the use of multi-hop, device-to-device communications over millimeter wave $(\mathrm{mmW})$ frequencies is studied for effective wearable communications. In particular, the problem of uplink communications is studied for a wearable network in which each wearable device seeks to form a multihop path over mmW to access a cellular base station, in order to overcome the high channel loss caused by $\mathrm{mmW}$ attenuation and blockage. To analyze the optimal selection of the uplink path, a network formation game is formulated between all wearable devices. In this game, each wearable device autonomously chooses the uplink path that maximizes its utility function that captures the tradeoff between rate, delay, and privacy. To solve this game, a novel algorithm that combines best response dynamics with mixed-strategy techniques is proposed to find the mixed Nash network, which corresponds to a stable uplink structure at which no wearable device can improve its utility by changing its network formation decision. Simulation results show that the proposed game approach improves the average utility per wearable device of over $14 \%$ and $78 \%$, respectively, compared with the direct transmission and the nearest next-hop schemes.
\end{abstract}

\section{INTRODUCTION}

The next decade will witness an unprecedented proliferation of wearable devices, such as smart watches, augmented-reality glasses, and fitness trackers, among others. These wearable devices can work collaboratively to form a body-surrounding network and provide the human wearers with comprehensive services, such as health monitoring, and interactive entertainment [1]. However, the success of the deployment of wearable devices is contingent upon enabling existing wireless networks to integrate a massive number of such devices. Given the congestion of sub- $6 \mathrm{GHz}$ frequency bands, recently, there has been significant interest [1], [2] in using millimeter wave ( $\mathrm{mmW}$ ) frequencies to enable large-scale wearable communications.

$\mathrm{MmW}$ frequencies encompass the bands ranging from 30 to $300 \mathrm{GHz}$. The rationale behind using $\mathrm{mmW}$ to deploy wearable networks stems from the availability of large bandwidth within the $\mathrm{mmW}$ frequency band. As such $\mathrm{mmW}$ frequencies are orthogonal to the commercial sub-6 GHz frequencies, $\mathrm{mmW}$ can support wearable communications, without degrading the performance of existing communication systems. Moreover, mmW bands can potentially support wearable de-

This research was supported by the U.S. National Science Foundation under Grant CNS-1513697, by the Office of Naval Research (ONR) under Grant N00014-15-1-2709, in part by the ERC Starting Grant 305123 MORE, and in part by the Academy of Finland project CARMA. vices with a larger bandwidth which can provide significant improvements in their transmission reliability.

However, communications over $\mathrm{mmW}$ frequencies face many challenges. The smaller wavelengths of $\mathrm{mmW}$, compared with the sub-6 GHz spectrum, will result in a faster signal degradation. In order to overcome the serious path loss, beamforming can be used to increase the channel gain of $\mathrm{mmW}$ transmissions. However, $\mathrm{mmW}$ is also highly sensitive to blockage from common objects, such as a wall and a human body, which will effectively lead to a serious signal attenuation. Several recent works [3]-[5] have proposed the use of multi-hop transmissions to improve the communication reliability over $\mathrm{mmW}$ in presence of the obstacles.

The authors in [3] consider a wireless personal area network (WPAN) and present a cross-layer model to study a multi-hop medium access control architecture at the $60 \mathrm{GHz}$ band. The authors in [4] propose a multi-hop selection metric, as well as a concurrent transmission scheme, to exploit the data rate of a mmW-based WPAN. The work in [5] studies a centralized multi-hop routing approach that maximizes the amount of traffic that circulates over the multi-hop links in a $\mathrm{mmW}$ based cellular network. However, these existing works [3][5] either focus on individual mmW links without providing a comprehensive analysis from a system level, or do not properly capture the unique characteristics of $\mathrm{mmW}$ communications, such as the sensitivity to blockage or their short transmission range.

The main contribution of this paper is a novel framework that enables wearable devices to autonomously establish a multi-hop network. We study the uplink communication scenario in which each wearable device aims to form a multihop path over mmW to access the base station (BS), so as to maximize its uplink quality-of-service (QoS), in terms of rate, delay, and privacy. We model the problem as a network formation game between wearable devices so as to analyze the optimal uplink transmissions. To solve this game, we propose an iterative algorithm, based on the best response dynamics and the mixed strategy approach, to find the mixed Nash equilibrium (NE), which corresponds to a stable network architecture. The proposed approach is shown to always find a mixed-Nash NE with low complexity. Simulation results show that the proposed game-theoretic approach significantly improves the average utility of uplink transmissions per wearable device, compared with a conventional direct-transmission 
scheme and a nearest next-hop approach.

The rest of the paper is organized as follows. Section II presents the system model. The network formation game formulation and solution are proposed in Section III. Simulation results are presented in Section IV. Finally, conclusions are drawn in Section V.

\section{SySTEM MODEL}

Consider the uplink of a wireless cellular network consisting of one BS and $M$ human users. Each user is equipped with one mobile phone and $L$ wearable devices (WDs). Let $m$ denote the BS, $\mathcal{I}$ be the set of all WDs, where $|\mathcal{I}|=M \times L$, and $\mathcal{J}$ be the set of all mobile phones. Then, the set of all communication devices in this system is $\mathcal{N}=\mathcal{I} \cup \mathcal{J} \cup\{m\}$. In this model, the WDs transmit data via $\mathrm{mmW}$ bands, while the cellular communications between the BS and mobile phones use the sub- $6 \mathrm{GHz}$ spectrum. However, we assume that the BS and phones are dual-mode and can use both the $\mathrm{mmW}$ and sub- $6 \mathrm{GHz}$ frequencies. Therefore, all communication devices in $\mathcal{N}$ can receive $\mathrm{mmW}$ signals. Furthermore, we assume that each WD has a dedicated $\mathrm{mmW}$ channel, through which it can transmit data directly to the BS. However, such direct communication may not be reliable, as the signal-to-noise-ratio (SNR) of mmW channels is highly susceptible to blockage. Therefore, in order to improve the uplink QoS, a WD can choose one device in $\mathcal{N}$ as its next hop to receive and forward its messages to the BS. The possible next hop can be any other phone or WD. Once the next hop is determined, each WD automatically inherits the uplink path of the next hop, and the uplink path of itself is determined. Naturally, the uplink path of some WD can be multi-hop.

To mathematically represent the architecture of the wearable network in the uplink, a directed graph $G=(\mathcal{N}, \mathcal{E})$ is introduced, where $\mathcal{N}$ is the set of all communication nodes, and $\mathcal{E}$ is the set of directed edges, which correspond to the uplink traffic flows. A directed link (edge) from device $i$ to $j$ is denoted by $(i, j)$, and $\mathcal{E}$ is the set of all existing links in $G$. Then, the path $p_{i}$ from a WD $i \in \mathcal{I}$ to the BS is defined as a sequence of nodes $i_{1}, i_{2}, \cdots, i_{K}\left(i_{k} \in \mathcal{N}\right)$ such that $i_{1}=i$ is the WD itself and $i_{K}=m$ is the BS. Since the choice of the next hop determines the uplink path, we denote the uplink path of WD $i$ with a given next hop $i_{2}$ as $p_{i}\left(i_{2}\right)$, where $p_{i}\left(i_{2}\right)=\left\{\left(i_{k}, i_{k+1}\right) \in \mathcal{E} \mid k=1, \cdots, K-1\right\}$. Note that, for each mobile phone $j \in \mathcal{J}$, the next hop is always the BS, therefore, its uplink is a one-hop path, denoted by $p_{j}(m)=\{(j, m)\}$. Next, we abbreviate the uplink path of device $i$ to be $p_{i}$ if its next hop is unconspicuous.

Fig. 1 shows an illustrative example of a wearable network within a cellular system. However, such a network architecture does not necessarily maximize the system performance, since blockage may occur over the $\mathrm{mmW}$ channel, which significantly decreases the QoS of the $\mathrm{mmW}$ transmission. In such a case, the involved WD has an incentive to improve the uplink communication by changing the current next hop to some other device experiencing a better multi-hop channel. Consequently, our goal is to study how each WD can determine the most

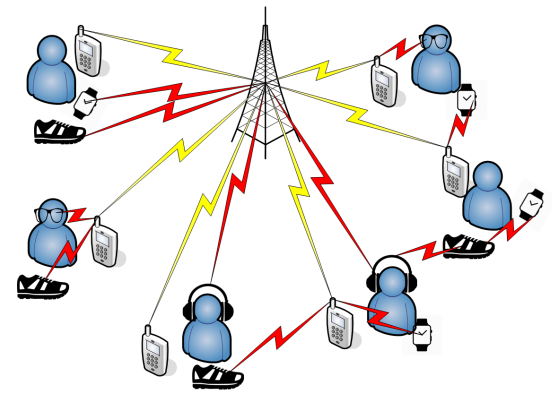

Fig. 1: An illustrative example of the uplink network, where the yellow links denote the cellular channels over sub-6 GHz frequency, and the red links are the wearable communications over $\mathrm{mmW}$.

efficient multi-hop path to transmit its data to the BS. However, prior to analyzing this network formation process, we need to define the QoS metrics, which each WD can use to evaluate an uplink path, considering three aspects: rate, delay, and privacy, as detailed next.

\section{A. Transmission Capacity}

Since emerging WDs, such as interactive augmented reality glasses, can require a high data rate [6], the communication capacity will be a significant parameter to evaluate the multihop transmission for future wearable networks.

1) Channel Model: The human body is modeled by a circle that represents the blockage area of the $\mathrm{mmW}$ communication links [6]. The most common statistical model, based on the real-world measurements in [7], describes the average path loss and fading value of a mmW link $(i, j)$ in $\mathrm{dB}$ as follows:

$$
\ell_{i j}=a_{i j}+b_{i j} \log _{10}\left(\left\|\boldsymbol{x}_{i}-\boldsymbol{x}_{j}\right\|\right)+n_{i j} \log _{10}\left(f_{i j}\right)+z_{i j}
$$

where $a_{i j}, b_{i j}$ and $n_{i j}$ are the path loss parameters for mmW communications, $\boldsymbol{x}_{i}$ and $\boldsymbol{x}_{j} \in \mathbb{R}^{2}$ represent the location of transmitter $i$ and receiver $j$ with $\|\cdot\|$ being their distance in meters, $f_{i j}$ denotes the carrier frequency of the link $(i, j)$, and $z_{i j}$ is the shadow fading term of mmW. For the lineof-sight and non-line-of-sight links over $\mathrm{mmW}$, different of parameters will be applied according to the link state. The typical parameter values are available in [7]. The linear value of such channel model is given as $h_{i j}=10^{-0.1 \ell_{i j}}$.

The path loss of the cellular communication links between mobile phones and the BS follows a similar model to (1), but with different parameters, which are available in [8].

2) Antenna Gain: In order to compensate for the high attenuation, mmW WDs will typically implement beamforming through directional antenna arrays [9]. For a mmW link $(i, j)$, where $i \in \mathcal{I}$, the overall antenna gain $g_{i j}\left(\boldsymbol{x}_{i}, \boldsymbol{x}_{j}\right)$ is a function of the locations of transmitter $i$ and receiver $j$ [1]. For tractability, here, we assume a perfect beam alignment between $\mathrm{mmW}$ transceivers [6]. Therefore, the function is simplified as $g_{i j}=g_{i} \cdot g_{j}$, where $g_{i}$ and $g_{j}$ are the main-lobe antenna gains of device $i$ and $j$. For the transceiver in the cellular communications, we assume the single-antenna devices with a constant gain. 
3) Achievable Transmission Rate: The channel capacity for a general wireless link $(i, j)$ can be given as:

$$
c_{i j}=w_{i j} \log _{2}\left(1+\frac{g_{i j} P_{i} h_{i j}}{w_{i j} N_{j}+I_{i j}}\right),
$$

where $w_{i j}$ is the bandwidth of link $(i, j), P_{i}$ denotes the transmission power of device $i, N_{j}$ is the noise power density, and $I_{i j}$ represents the interference at receiver $j$. The bandwidth for each mmW link is equal to the total available bandwidth divided by the number of WDs. Here, considering the impenetrability of $\mathrm{mmW}$ over obstacles and the dedicated channel for each WD, we assume that $I_{i j}=0, \forall i \in \mathcal{I}$. However, for the cellular communication links, the interference $I_{i j}, i \in \mathcal{J}, j=m$ is equal to the sum of the received power of co-channel signals from the adjacent cells.

Further, we assume that, for each device in $\mathcal{N}$, the transmitting and receiving antennas are separated. Then, given (2), the achievable end-to-end data rate of an uplink path $p_{i}$ can be defined as the minimum capacity among all the hops along this path. Let $K_{i}=\left|p_{i}\right|$ be the number of nodes on path $p_{i}$, the average uplink rate, which WD $i$ can achieve over path $p_{i}$, will be [10]:

$$
c_{i}\left(p_{i}\right)=\min _{\left(i_{k}, i_{k+1}\right) \in p_{i}} c_{i_{k} i_{k+1}}, \forall k=1, \cdots, K_{i}-1 .
$$

\section{B. Latency}

One key metric for multi-hop transmission is the latency due to the multiple hops on the transmission path from the source to the destination, as well as from the potential buffering at each node on the path [11].

Here, we model each device $i \in \mathcal{N}$ as a data source, which sends packet to its next hop following a Poisson process with an average rate of $\lambda_{i}$. At each node, an $M / D / 1$ queuing [12] model is adopted, and the incoming packets are stored and transmitted in a first-in-first-out fashion. Let $\tau_{i j}$ be the latency, experienced by a packet of $B$ bits, on a general link $(i, j) \in \mathcal{E}$. As shown in [12], the average delay will be:

$$
\tau_{i j}(G)= \begin{cases}\frac{\lambda_{i j}}{2 \mu_{i j}\left(\mu_{i j}-\lambda_{i j}\right)}+\frac{1}{\mu_{i j}}, & \mu_{i j}>\lambda_{i j}, \\ \infty, & \mu_{i j} \leq \lambda_{i j},\end{cases}
$$

where $\lambda_{i j}=\sum_{(i, j) \in p_{k}, k \in \mathcal{N}} \lambda_{k}$ is the amount of traffic load flowing on the link $(i, j)$, which is equal to the sum of data from the nodes $k \in \mathcal{N}$, whose uplink path $p_{k}$ includes $(i, j)$. Here, $1 / \mu_{i j}=B / c_{i j}$ is the transmission delay over link $(i, j)$. If $\mu_{i j}>\lambda_{i j}$, which indicates the arrival rate of data is smaller than the capacity of the link, the term $\lambda_{i j} /\left(2 \mu_{i j}\left(\mu_{i j}-\lambda_{i j}\right)\right)$ captures the waiting time in the queue of node $i$ before the arriving packet can be transmitted. Therefore, the first case in (4) represents the time interval for a packet to pass through link $(i, j)$, when $\mu_{i j}>\lambda_{i j}$. However, for $\mu_{i j} \leq \lambda_{i j}$, the transmission over $(i, j)$ will be congested, and to go through this link, a new upcoming packet will experience a large latency, which, for simplicity, is considered infinite. Note that, the traffic load of each link $(i, j)$ is the sum of the traffic over all lower branches of node $i$. Therefore, the latency $\tau_{i j}$

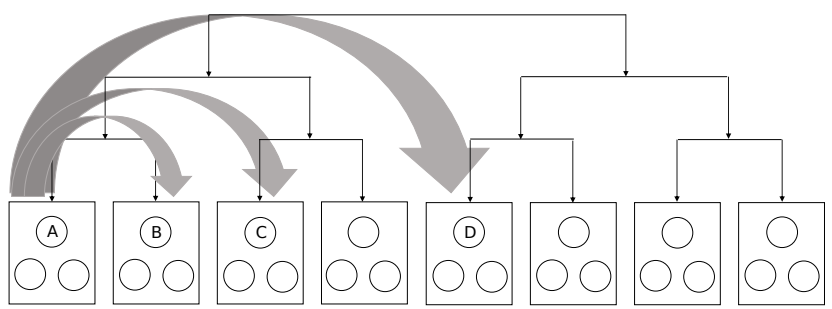

Fig. 2: The binary tree model of the society distance. For example, the social distance between user A and user B is one, the distance between $\mathrm{A}$ and $\mathrm{C}$ is two and the distance between $\mathrm{A}$ and $\mathrm{D}$ is three.

depends not only on the uplink path of WD $i$, but also on all other nodes $\left\{k \mid(i, j) \in p_{k}\right\}$ that select $i$ as an intermediate node on their paths. Therefore, $\tau_{i j}$ should be a function of a subnetwork of $G$, instead of only $p_{i}$.

Given the latency of each link $(i, j) \in \mathcal{E}$, the latency experienced by a device $i$ to send a packet over an uplink path $p_{i}$ will be given by:

$$
\tau_{i}(G)=\sum_{\left(i_{k}, i_{k+1}\right) \in p_{i}} \tau_{i_{k} i_{k+1}}, \forall k=1, \cdots, K_{i}-1 .
$$

\section{Privacy Consideration}

Given that WDs are typically affixed to humans, several privacy concerns may arise during multi-hop communications. In order to get a better service, each WD can choose other nodes to forward its messages to the BS. However, as the intermediate nodes on the uplink path could tap into the content, the privacy of the user may be compromised. To avoid any disclosure of private information, in addition to the use of standard encryption techniques, each WD will have a motivation to choose the devices belonging to a trustworthy person to form an uplink path, instead of those that belongs to less trustworthy users.

To capture such privacy concerns, we introduce a binary tree model [13] to formulate the distance of affinity between different users, as well as their devices. Note that, the social tree model is independent from the communication architecture of the wearable network. As shown in Fig. 2, for any two given device $i$ and $j$ located on the leaves of the binary tree, the affinity distance $d_{i j}$ is defined as the number of layers to ascend, so that device $i$ and $j$ can find their first common bifurcation, which is defined as the first common generic node on the binary tree. Then, the privacy parameter for WD $i$ that uses a certain path $p_{i}$ to connect to the $\mathrm{BS}$ is defined as

$$
v_{i}\left(p_{i}\right)=e^{-\sum_{\left(i_{k}, i_{k+1}\right) \in p_{i}} d_{i_{k} i_{k+1}}}, \forall k=1, \cdots, K_{i}-1 .
$$

\section{Problem Formulation and Solution}

Given the network introduced in Section II, the main objective is to study how each WD chooses the next hop to form a multi-hop uplink towards the BS which optimizes the transmission QoS that is function of rate, latency, and privacy.

Note that, the change of the next hop by any WD will not only vary its uplink QoS, but will also impact many other devices, due to the reallocation of traffic load in the network. In order to model the interactions between WDs, the framework of network formation games can be used, as it provides a set of 
tools to analyze how independent decision makers can interact to form a suitable connected graph [11]. Here, we note that there has been some recent works on network formation games in wireless networks, such as in [10] and [11]. However, these works study the traditional cellular communications, while our work deals with $\mathrm{mmW}$ communications for wearable devices within the emerging Internet of things (IoT) ecosystem.

\section{A. Network Formation Game}

We formulate the network formation problem as a noncooperative game $\mathcal{G}=\left(\mathcal{I},\left\{\mathcal{S}_{i}\right\}_{i \in \mathcal{I}},\left\{u_{i}(G)\right\}_{i \in \mathcal{I}}\right)$, in which $\mathcal{I}$ is the set of players which are the WDs, $\mathcal{S}_{i}$ is the strategy space for each player $i$, and $u_{i}(G)$ is the utility function.

1) Strategy Space: The strategy space $\mathcal{S}_{i}$ of each player $i \in \mathcal{I}$ includes all the feasible devices that WD $i$ can choose to form an uplink path. Each strategy $s_{i} \in \mathcal{S}_{i}$ will cause a sequence of operations on the links [10]. For example, by choosing the strategy $s_{i}=(i, j)$, WD $i$ will first break the link $\left(i, j^{o}\right)$ with its former next hop $j^{o}$, and then, build a new link $(i, j)$. For notational simplicity, we denote the network structure as $G_{(i, j)}$ to indicate the next hop of WD $i$.

However, whenever a device $j$ accepts a new link, due to the increased traffic load, its uplink delay will increase. Therefore, we assume that each device can reject a device-todevice (D2D) transmission request, if the formation of such a link will degrade its transmission. Consequently, the feasible strategy set of a WD $i$ is defined as the set of devices who are willing to accept the connection from $i$, denoted by

$$
\mathcal{S}_{i}=\left\{(i, j) \mid \tau_{j}\left(G_{(i, j)}\right)-\tau_{j}\left(G_{\left(i, j^{\circ}\right)}\right)<\varepsilon_{j}, j \in \mathcal{N}\right\},
$$

where $\varepsilon_{j} \in \mathbb{R}^{+}$is a small positive amount, which denotes the maximum delay increase that device $j$ can tolerate by accepting link $(i, j)$.

As long as each WD $i \in \mathcal{I}$ has chosen its next hop $s_{i} \in \mathcal{S}_{i}$, the edge set $\mathcal{E}$ of the network graph is determined as $\mathcal{E}=\left\{s_{1}, s_{2}, \cdots, s_{I}, s_{I+1}, \cdots, s_{N-1}\right\}$, where $\left\{s_{1}, s_{2}, \cdots, s_{I}\right\} \in\left\{\mathcal{S}_{i}\right\}_{i \in \mathcal{I}}$ is the strategy profile of all players in $\mathcal{I}$, and $\left\{s_{I+1}, \cdots, s_{N-1}\right\}=\{(I+1, m), \cdots,(N-1, m)\}$ is the uplink set of mobile phones, which is unaltered.

2) Utility Function: The utility function should reflect the incentive of each WD to form a multi-hop path, by taking into account the data rate, latency, and privacy concern to evaluate the uplink transmission. To this end, we introduce the concept of the weighted power of a system [14], where the power of a network is defined by the ratio of the throughput to the response time of the uplink path, weighted by the privacy parameter. The weighted power emphasizes the tradeoff between three metrics. Then, the utility function of WD $i$ that chooses node $j$ as its next hop will be:

$$
u_{i}\left(G_{(i, j)}\right)=v_{i}\left(p_{i}(j)\right)^{\gamma_{i}} \cdot \frac{c_{i}\left(p_{i}(j)\right)^{\alpha_{i}}}{\tau_{i}\left(G_{(i, j)}\right)^{\beta_{i}}},
$$

where $c_{i}, \tau_{i}$ and $v_{i}$ are the rate, delay and privacy parameter of path $p_{i}(j)$, respectively, and $\alpha_{i}, \beta_{i}, \gamma_{i} \in \mathbb{R}^{+}$are the weights, which capture the importance of each of the three metrics that WD $i$ will consider when evaluating the uplink QoS of $p_{i}(j)$.
Thus, the objective of each WD $i \in \mathcal{I}$ is to compare the transmission quality of each uplink path $p_{i}(j),(i, j) \in \mathcal{S}_{i}$ by calculating the utility $u_{i}\left(G_{(i, j)}\right)$, and choose the optimal hop.

\section{B. Game Solution}

In a noncooperative game, each WD aims to maximize the uplink QoS of itself, without considering other players. To this end, each device can update its next hop, according to the change of the network architecture, to improve the payoff. However, such an update may impact the utilities of other players, which can motivate some WDs to revise their uplink paths to get a better utility, and, hence, the network structure may change repeatedly. Therefore, a reasonable solution for the game must identify a stable state, at which no WD has an incentive to change its current path. Consequently, we introduce the concept of the Nash network as the solution to the network formation game, defined next.

Definition 1: A Nash network of the network formation game $\mathcal{G}=\left(\mathcal{I},\left\{\mathcal{S}_{i}\right\}_{i \in \mathcal{I}},\left\{u_{i}(G)\right\}_{i \in \mathcal{I}}\right)$ defines a network graph $G^{*}\left(\mathcal{N}, \mathcal{E}^{*}\right)$, where the edge set $\mathcal{E}^{*}=$ $\left\{s_{1}^{*}, s_{2}^{*}, \cdots, s_{I}^{*}, s_{I+1}, \cdots, s_{N-1}\right\}$ guarantees that for all $i \in \mathcal{I}$, the following holds:

$$
u_{i}\left(G_{\left(s_{i}^{\prime}, \mathcal{E}_{-i}^{*}\right)}\right) \leq u_{i}\left(G_{\left(s_{i}^{*}, \mathcal{E}_{-i}^{*}\right)}^{*}\right), \forall s_{i}^{\prime} \in \mathcal{S}_{i},
$$

where $\mathcal{E}_{-i}^{*}=\left\{s_{1}^{*}, \cdots, s_{i-1}^{*}, s_{i+1}^{*}, \cdots, s_{I}^{*}, s_{I+1}, \cdots, s_{N-1}\right\}$ is the profile of the next hops for all players in $\mathcal{I}$, except $i$. The Nash network is the concept of Nash equilibrium applied to a network formation game [11]. In essence, a Nash network is a stable graph $G^{*}$, where no WD can improve its uplink QoS, by unilaterally changing its next hop from the current $j^{*}$, where $s_{i}^{*}=\left(i, j^{*}\right)$, to any others $j^{\prime}$ in its feasible set $\mathcal{S}_{i}$, given that the choices $s_{-i}^{*}$ of all the other WDs are fixed.

The aforementioned solution, where each player chooses a deterministic strategy to form the uplink path, is called a pure-strategy NE. However, for a general game, the purestrategy NE may not exist. To guarantee a stable output, a mixed strategy approach can be used, since the mixed-strategy $\mathrm{NE}$ always exists [15]. In a mixed-strategy case, WD $i$ chooses a probability distribution $\mathbb{P}\left(\mathcal{S}_{i}\right)$ over all feasible next-hop choices. Then, every time a packet needs to be delivered, the next hop will be chosen from $\mathcal{S}_{i}$ according to $\mathbb{P}\left(\mathcal{S}_{i}\right)$. For this mixed-strategy case, the expected utility for a player $i$ will be given by:

$$
\bar{u}_{i}\left(\left\{\mathbb{P}\left(\mathcal{S}_{j}\right)\right\}_{j \in \mathcal{I}}\right)=\sum_{s_{i} \in \mathcal{S}_{i}}\left(\prod_{j=1}^{I} \mathbb{P}\left(\mathcal{S}_{j}\right)\right) u_{i}\left(G_{\left(s_{i}, \boldsymbol{s}_{-i}\right)}\right) .
$$

Consequently, the mixed-strategy NE will be defined as a probability distribution $\left\{\mathbb{P}^{*}\left(\mathcal{S}_{i}\right)\right\}_{i \in \mathcal{I}}$, at which no player can improve its expected utility by unilaterally changing its mixed strategy $\mathbb{P}^{*}\left(\mathcal{S}_{i}\right)$. Although the existence of the mixed NE is guaranteed, the complexity to find a mixed $\mathrm{NE}$ can be higher than the pure strategy case. Next, to solve the network formation game $\mathcal{G}$ efficiently, we propose a novel, low-complexity algorithm. 


\section{Network Formation Algorithm}

The network formation process starts with an initial tree structure, in which each WD connects to the BS directly. Then, WDs sequentially ${ }^{1}$ apply the following actions.

1) Information Collection: When a WD $i$ must take an action, it will first send a D2D transmission request, which contains the necessary information used for uplink estimation such as the location and the traffic load, via a common $\mathrm{mmW}$ channel. Due to blockage, the $\mathrm{mmW}$ broadcast can only cover a small range. However, given that WD $i$ must have a good channel gain with any choice of a next hop, the nodes which cannot receive the signal will naturally not be feasible to act as the next hop of WD $i$. After receiving the broadcast, the feasible devices calculate the utility from accepting such a D2D link from WD $i$. If the increase in delay is intolerable, the request will be ignored, otherwise WD $i$ will receive a response from the feasible device in question. This response will contain the next hop's information and the utility.

2) Best Response: After receiving the response, WD $i$ will accept as its next hop, the WD that has the highest payoff, if such uplink is better than the current path. Otherwise, if no node replies $\left(\mathcal{S}_{i}=\emptyset\right)$, or no responding node can offer a better uplink transmission, WD $i$ will keep its current path. Then, WD $i$ informs the BS whether it makes any change, and releases the broadcast channel for other WDs to make the D2D request.

3) Pure Nash Network: The process continues until one of the following conditions is satisfied: (i) no WD can improve its utility by deviating from the current uplink path; (ii) the BS realizes that the current network structure $G$ has been visited for more than $\rho \in \mathbb{N}^{+}$times. If condition (i) occurs, the game ends with a pure Nash network. If condition (ii) occurs, the network architecture may cycle between certain patterns periodically, and the best response dynamics fail to converge to the pure Nash network. Then, some of WDs can move to use mixed strategies.

4) Mixed Nash Network: According to the network evolution, the set of WDs $\mathcal{I}$ will be divided into two disjoint subsets $\mathcal{I}_{1} \cup \mathcal{I}_{2}$, where $\mathcal{I}_{1}$ contains the loop nodes which keep changing their next-hop choices at each iteration, and $\mathcal{I}_{2}$ is the set of stable WDs that maintain their current next hops and never deviate after a few iterations. Then, the subnetwork formed by stable nodes in $\mathcal{I}_{2}$ is already a stable network, while the loop WDs in $\mathcal{I}_{1}$ will start to apply the mixed strategies to find the mixed NE through a centralized approach [15].

5) Hybrid Solution: The hybrid Nash network $G^{H}$, which is formed by the mixed Nash subnetwork generated by loop players in $\mathcal{I}_{1}$ and the pure Nash subnetwork formed by stable players in $\mathcal{I}_{2}$, is the final output of the network formation game $\mathcal{G}$.

However, after the mixed strategy game between players in $\mathcal{I}_{1}$, one cannot guarantee that the pure strategy for each node in $\mathcal{I}_{2}$ is still the best response strategy in the final network

\footnotetext{
${ }^{1}$ The sequence depends on the order in which WDs make the D2D transmission requests.
}

structure $G^{H}$. Therefore, more analysis is needed to prove the stability of the final game output, as shown next.

Theorem 1: The hybrid Nash network $G^{H}$ is a mixed Nash equilibrium.

Proof: If the best response dynamics fails to converge to a pure Nash network, the network structure will move between certain patterns periodically, and we denote the set of the network structures that appear within one period as $G^{W}=\left\{G^{1}, G^{2}, \cdots, G^{w}\right\}$. Then, the mixed-strategy game is played between wearable nodes in $\mathcal{I}_{1}$. The output of the mixed-strategy game is a probability distribution $\Xi^{\mathcal{I}_{1}}$ over all network structures in $G^{W}$, where $\Xi^{\mathcal{I}_{1}}=\left\{\xi_{1}, \xi_{2}, \cdots, \xi_{w}\right\}$, and $\xi_{q}$ denotes the probability that the network structure $G^{q}$ is selected when some node in $\mathcal{I}_{1}$ delivers a packet to the BS. Although $\Xi^{\mathcal{I}_{1}}$ defines a stable structure of the subnet, formed by nodes of $\mathcal{I}_{1}$, however, for the nodes of $\mathcal{I}_{2}$, we need to prove their stability after the mixed game.

Note that, during the network loop $G^{W}$, in best response dynamics, no node in $\mathcal{I}_{2}$ changes its next-hop choice, which means the following inequality (11) holds for each device $i \in$ $\mathcal{I}_{2}$ in each network structure $G^{q} \in G^{W}$.

$$
u_{i}\left(G_{(i, j)}^{q}\right) \geq u_{i}\left(G_{\left(i, j^{\prime}\right)}^{q}\right), \forall j^{\prime} \in \mathcal{S}_{i} .
$$

Then, after the mixed game, given the probability distribution $\Xi^{\mathcal{I}_{1}}$, the expected utility that each device in $\mathcal{I}_{2}$ gets from the hybrid network $G^{H}$ will be

$$
\bar{u}_{i}\left(G_{(i, j)}^{H}\right)=\sum_{q=1}^{w} \xi_{q} u_{i}\left(G_{(i, j)}^{q}\right) .
$$

Then, by substituting (11) into (12), we get

$$
\bar{u}_{i}\left(G_{(i, j)}^{H}\right) \geq \sum_{q=1}^{w} \xi_{q} u_{i}\left(G_{\left(i, j^{\prime}\right)}^{q}\right)=\bar{u}_{i}\left(G_{\left(i, j^{\prime}\right)}^{H}\right), \forall j^{\prime} \in \mathcal{S}_{i} .
$$

Therefore, the hybrid Nash network $G^{H}$ is actually a mixed $\mathrm{NE}$, in which the players in $\mathcal{I}_{1}$ adopt mixed strategies and the players in $\mathcal{I}_{2}$ have pure strategies.

Such a hybrid approach can be applied in any noncooperative game to find the mixed NE. In the first phase, the best response dynamics is applied to find the pure NE for some players; then, the mixed strategy game is played between the other players to find a mixed NE. This approach can always find a mixed NE, and the computations to find the mixed NE are reduced by decreasing the number of players that engage in mixed strategies through the first pure-strategy game.

\section{Simulation Results And Analysis}

For simulations, a wearable network that uses the $60 \mathrm{GHz}$ frequency band is deployed in a hexagonal indoor area with a radius of 6 meters, and the BS is located in the center. In this network, each human user has four wearable devices and one phone. The total available bandwidth for $\mathrm{mmW}$ communications is $1 \mathrm{GHz}$. For cellular communications, mobiles transmit data to the BS over $2 \mathrm{GHz}$. The channel parameters of $\mathrm{mmW}$ and cellular transmissions are chosen based on [7] 


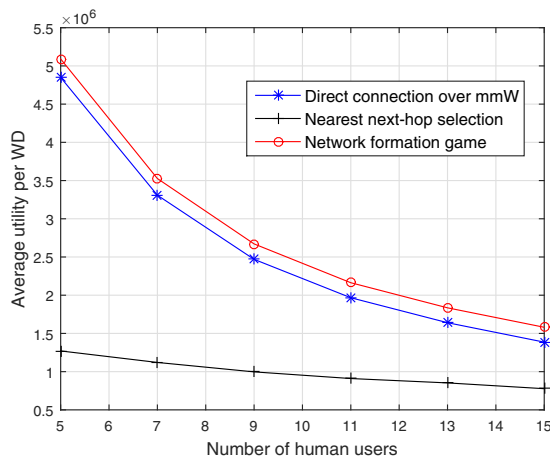

(a)

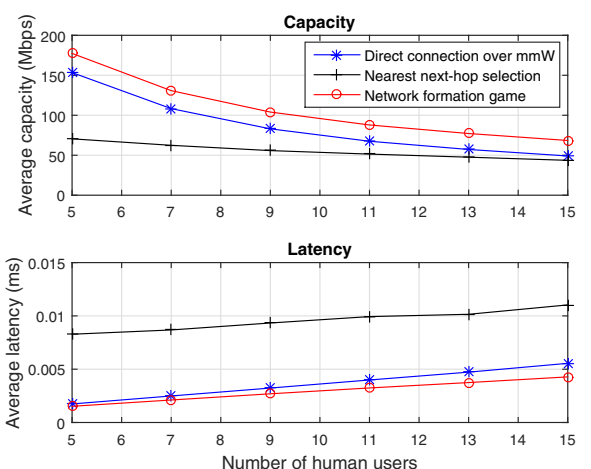

(b)

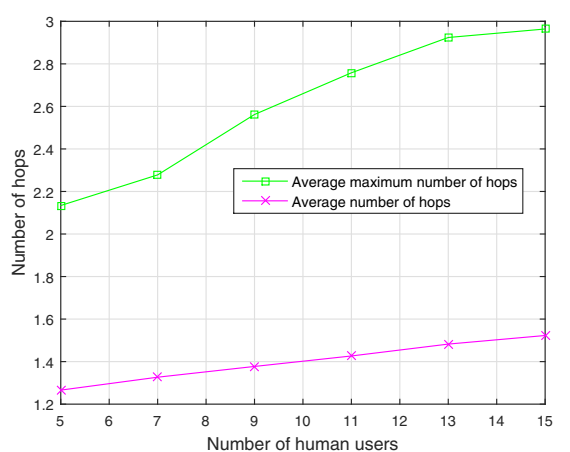

(c)

Fig. 3: (a) The proposed game approach yields a better uplink QoS, compared with the direct transmission scheme and the nearest next-hop approach. (b) The average capacities of the three approaches decreases as the number of users increases, while the average latency increases linearly. (c) The average number and the average maximum number of hops will both increase as the number of WD increases.

and [8], respectively. In all simulations, the average number of iterations for convergence is around three.

Fig. 3a shows the average utility per wearable device, as the number of human users increases from five to fifteen. Here, the number of players (i.e. wearable devices) increases from 20 to 60 . The weight vector of rate, latency, and privacy is set to be $(0.1,0.1,1)$. We assess the performance of the formed uplink network structure by comparing it with a direct-transmission scheme, and a nearest next-hop approach. Fig. 3a first shows that, as the number of WDs increases, the average utilities of all three methods decrease. The utility drop is caused by the reduction in the $\mathrm{mmW}$ bandwidth per WD. Also, as the network becomes more crowded, the increase of traffic load results in a larger latency. Fig. 3a shows that the proposed game yields a significant advantage, in terms of average utility, up to $14 \%$ and $78 \%$, over the direct transmission scheme and the nearest next-hop method, respectively.

Fig. 3b shows the average capacity and latency per WD, as the number of users increases. First, we can see that the game-theoretic approach yields an advantage over the direct transmission and the nearest next-hop schemes of up to $40 \%$ and $70 \%$, respectively. Also, the game-theoretic approach decreases the uplink latency by up to $23 \%$ and $300 \%$, compared with the other two methods. Furthermore, the average capacities of all mentioned approaches will decrease as the number of users increases, while the latencies increase in a linear way.

Fig. $3 c$ gives the average hops and the average maximum number of hops in the final network structures. As the number of wearable devices increases, both the average and the maximum number of hops increases, but the increase rate is very slow. Given that the weights of the metrics are dominated by latency, each wearable device will prefer a shorter path to access the BS to guarantee a lower delay. Therefore, on average, the number of hops remains below three.

\section{CONCLUSION}

In this paper, we studied the problem of distributed network formation among wearable devices in a mmW network. We have cast the problem as a network formation game in which each WD seeks to find the uplink path which maximizes the QoS tradeoff between rate, delay, and privacy. To solve this game, we have introduced a novel algorithm that combines best response dynamics with mixed-strategy techniques. We have shown that this algorithm exhibits low complexity and can reach a mixed-strategy Nash network. Simulation results have shown that the proposed game can significantly improve the average utility per WD, compared with the direct transmission and the nearest next-hop schemes.

\section{REFERENCES}

[1] Q. Zhang, W. Saad, M. Bennis, and M. Debbah, "Quantum game theory for beam alignment in millimeter wave device-to-device communications," in Proc. of the IEEE Global Communications Conference, Washington, DC, USA, Dec. 2016.

[2] K. Venugopal and J. R. W. Heath, "Location based performance model for indoor mmwave wearable communication," in Proc. IEEE Int. Conf. on Commun. (ICC), Kuala Lumpur, Malaysia, May 2016.

[3] S. Singh, F. Ziliotto, U. Madhow, E. M. Belding, and M. J. W. Rodwell, "Millimeter wave WPAN: Cross-layer modeling and multihop architecture," in Proc. IEEE INFOCOM 2007, Anchorage, AK, May 2007.

[4] J. Qiao, L. Cai, X. Shen, and J. W. Mark, "Enabling multi-hop concurrent transmissions in $60 \mathrm{GHz}$ wireless personal area networks," IEEE Trans. Wireless Commun., vol. 10, no. 11, Nov. 2011.

[5] J. Kim and A. F. Molisch, "Quality-aware millimeter-wave device-todevice multi-hop routing for $5 \mathrm{G}$ cellular networks," in Proc. IEEE Int. Conf. on Commun. (ICC), Sydney, Australia, Jun. 2014.

[6] K. Venugopal and R. W. Heath, "Millimeter wave networked wearables in dense indoor environments," IEEE Access, vol. 4, pp. 1205-1221, Mar. 2016.

[7] E. Perahia, C. Cordeiro, M. Park, and L. L. Yang, "IEEE 802.11 ad: Defining the next generation multi-Gbps Wi-Fi," in IEEE Consumer Communications and Networking Conference, 2010, pp. 1-5.

[8] G. Piro, L. A. Grieco, G. Boggia, F. Capozzi, and P. Camarda, "Simulating LTE cellular systems: An open-source framework," IEEE Trans. Veh. Technol., vol. 60, no. 2, Feb. 2011.

[9] O. Semiari, W. Saad, and M. Bennis, "Joint millimeter wave and microwave resources allocation in cellular networks with dual-mode base stations," IEEE Transactions on Wireless Communications, 2017.

[10] W. Saad, X. Zhou, B. Maham, T. Basar, and H. V. Poor, "Tree formation with physical layer security considerations in wireless multihop networks," IEEE Transactions on Wireless Communications, vol. 11, no. 11 , pp. 3980-3991, 2012.

[11] W. Saad, Ż. Han, T. Başar, M. Debbah, and A. Hjørungnes, "Network formation ,games among relay stations in next generation wireless networks," IEEE Trans. Commun., vol. 59, no. 9, pp. 2528-2542, Sep. 2011.

[12] D. Bertsekas and R. Gallager, Data networks. New Jersey, USA: Prentice Hall, Mar. 1992.

[13] A. Ludu, Boundaries of a Complex World. Springer-Verlag, Apr. 2014.

[14] L. Kleinrock, "Power and deterministic rules of thumb for probabilistic problems in computer communications," in Proc. Int. Conf. on Communications, Boston, USA, Jun. 1979.

[15] G. Bacci, S. Lasaulce, W. Saad, and L. Sanguinetti, "Game theory for networks: A tutorial on game-theoretic tools for emerging signal processing applications," IEEE Signal Process. Mag., vol. 33, pp. 94 119, Jan. 2016 